\title{
Tutta la gestione medica della calcolosi in una metanalisi? No grazie
}

\author{
Commento all'articolo: "Medical management to prevent recurrent nephrolithiasis in adults: \\ a systematic review for an American College of Physicians Clinical Guideline"
}

\section{Martino Marangella}

\author{
Consulente A.O. Ordine Mauriziano, S.C. Nefrologia e Dialisi, A.O Ordine Mauriziano di Torino, Torino
}

Questa metanalisi sulla prevenzione della nefrolitiasi recidivante nell'adulto (1) si caratterizza certo per accutarezza, rigore, ma anche coraggio.

L'introduzione sottolinea il grande impatto socio-economico della patologia, documentandolo con rimandi bibliografici aggiornati e precisi.

Il processo di estrazione, commento e valutazione degli studi RCT è stato impostato con il massimo scrupolo.

Gli Autori, per lo più di estrazione epidemiologica, si sono mossi con l'ottimismo dei non addetti ai lavori, non mettendo in conto il rischio di ritrovarsi con pochi studi randomizzati, a volte di bassa qualità, con pochi pazienti arruolati. Alla fine, per nessuno dei topics considerati sono emerse evidenze di forza adeguata.

Da "vecchio" frequentatore della calcolosi renale, non sono affatto stupito dei risultati di questa metanalisi.

Ma la domanda cui cercherò di rispondere è la seguente: gli RCT sono in grado di dare risposte basate sull'evidenza alle problematiche della gestione medica della nefrolitiasi?

Ovviamente tutti siamo capaci di disegnare un RCT, su un qualche aspetto della calcolosi di potenza adeguata a darci risposte. Ma in pratica questo cozza contro oggettive difficoltà:

a. la calcolosi è patologia cronica ma spesso ad andamento ciclico, difficilmente prevedibile;

b. la durata dei follow-up deve pertanto essere abbastanza lunga, direi non meno di 3 anni;

c. negli studi di intervento e sul lungo periodo, è molto difficile ottenere una buona compliance e i molti dropout, fino al 30-50\%, costringono ad ampliare il numero dei pazienti arruolati;

d. le recenti procedure urologiche non invasive hanno ridotto presso pazienti e medici non addetti l'appeal della gestione medica; e. infine, ma non per importanza, è quasi impossibile trovare risorse finanziarie fra i privati, e si può solo confidare nel sempre meno percorribile ricorso al pubblico.

Ciò premesso, vorrei discutere in maggior dettaglio alcuni degli items riportati nel lavoro.

a. I liquidi: solo due RCT riportati, entrambi di qualità medio-bassa. Non stupisce quanto sopra, specie se si pensa quanto sia difficile far mantenere volumi urinari stabilmente elevati ai pazienti. Nella nostra esperienza siamo riusciti nell'intento ma solo grazie al fatto che un'azienda aveva fornito gratuitamente e per 3 mesi, tre diversi tipi di acque ai pazienti arruolati (2). Inoltre, spesso i pazienti bevono ben più del solito in previsione di visite mediche o esami di laboratorio e questo inficia l'attendibilità dei dati.

b. La dieta: tre soli RCT hanno valutato l'impatto della dieta sulla calcolosi, sia nei singoli componenti che nella struttura complessiva. Anche qui i risultati sono inconcludenti e le evidenze insufficienti. Gli studi considerati non prevedevano controlli di compliance con dati oggettivi come la biochimica urinaria, il numero di pazienti non era adeguato, la durata del follow-up non abbastanza lunga, il tasso di recidive nei due bracci a volte basso, i fattori dietetici da controllare molteplici. Dobbiamo per questo concludere che la dieta non serve? Meglio dire che la potenza statistica dei tre RCT riportati era insufficiente.

c. I farmaci: la letteratura ci ha consegnato ad oggi RCT sui tiazidici, sul citrato e su allopurinolo. Qui emerge una discreta evidenza che, a parità di altre condizioni, sia i tiazidici, che i sali di citrato di potassio e magnesio, offrono un modesto ma significativo vantaggio in termini di prevenzione. Lo stesso vale per l'allopurinolo ma solo nei pazienti con calcolosi calcica associata ad iperuricuria. Tuttavia, dai pochi studi disponibili non siamo in grado di stabilire quali dosaggi siano necessari, se i vari sali di citrato siano equivalenti nella efficacia, quale dei farmaci sia preferibile, se vi siano effetti aggiuntivi nella associazione. Inoltre, prendiamo atto dell'atteso fallimento dell'acido acetoidrossamico nella calcolosi da infezione. Come pensare di opporsi con un farmaco alla produzione di ammonio derivante da un substrato così abbondante quale l'urea?

d. Biochimica urinaria: i risultati del tutto deludenti di correlazione fra biochimica e clinica non devono essere considerati sorprendenti. A parte l'arruolamento scarso nei pochi RCT disponibili, la biochimica urinaria presenta molti limiti:

a. Lo stone clinic effect altera i dati basali (3), specie se acquisiti in Centri specialistici.

b. Non sempre il timing degli esami è corretto, essendo spesso fatti a ridosso di un episodio acuto (4).

c. Le misurazioni vengono in genere effettuate su urine di 24 ore che esprimono una media di quanto avviene in quel giorno, ma 
non informa sull'andamento circadiano del rischio litogeno.

d. Ha poco senso predire o valutare l'andamento clinico basandosi su un singolo parametro. Sarebbe opportuno almeno stimare lo stato di saturazione.

e. Fra gli inibitori solo il citrato ed il magnesio compaiono nella lista degli esami di calcolosi. Di questi possiamo stimare l'effetto termodinamico, ma non quello, forse più importante, di tipo cinetico.

f. I profili metabolici e fisico-chimici si distribuiscono con un pattern continuo da cui un ampio overlapping fra gruppi di pazienti e fra pazienti e normali.

Possiamo dunque fare a meno della valutazione metabolica dei pazienti nel basale e nel follow-up?

Direi di no, perché gli esami di laboratorio:

a. selezionano i pazienti più complianti e li motivano a proseguire il follow-up medico;

b. informano sul contenuto effettivo della dieta in relazione ad apporto di proteine, totali ed animali, di sale, di calcio;

c. documentano il livello di aderenza dei pazienti alle prescrizioni;

d. individuano le calcolosi secondarie ad altre patologie;

e. orientano sulla natura della calcolosi nei molti casi in cui non si disponga di un esame chimico di calcoli o frammenti;

f. assistono nella scelta di provvedimenti che rispondano ad una impostazione olistica della cura, che tenga conto di fattori di rischio o patologie associate.

Dunque io continuerò a prescrivere esami di laboratorio aspettandomi da questi solo quello che sono in grado di dare. Nulla di più. Vorrei sottolineare come venga posta poca enfasi sulla composizione del calcolo o dei frammenti. Quante volte, in ambito medico, abbiamo diagnosticato una calcolosi urica o cistinica dopo che il paziente ha subito molteplici procedure urologiche? Sappiamo quanto il management medico possa essere cruciale sugli esiti di queste calcolosi. Le evidenze biochimiche e fisicochimiche in favore del trattamento sono così forti da non farci sentire la mancanza di RCT sulla prevenzione di questi calcoli. S'è mai fatto un RCT sull'uso del paracadute?

Per concludere, anche se le evidenze dei benefici del management medico non sono sostenute da RCT convincenti, l'esperienza clinica insegna che si può modificare in senso positivo l'andamento di una nefrolitiasi recidivante e severa. L'obiettivo da perseguire è quello di una riduzione stabile e significativa della saturazione urinaria e questa la si ottiene meglio con una terapia mirata sulle alterazioni metaboliche che favoriscono la soprasaturazione.

E poi la chiave del successo è la capacità del medico di ottenere una duratura aderenza a prescrizioni che dovranno essere semplici applicabili e personalizzate, dopo avere scrupolosamente edotto il paziente sulle potenziali conseguenze della calcolosi sulla sua qualità di vita.

Dichiarazione di conflitto di interesse: L'Autore dichiara di non avere conflitto di interessi.

Contributi economici agli Autori: L'Autore dichiara di non avere ricevuto sponsorizzazioni economiche per la preparazione dell'articolo.

Indirizzo dell'Autore:

Dr. Martino Marangella

Consulente A.O. Ordine Mauriziano

S.C. Nefrologia e Dialisi

Largo Turati 62

10128 Torino

mmarangella@alice.it

\section{Bibliografia}

1. Fink HA, Wilt TJ, Eidman KE, et al. Medical management to prevention recurrent nephrolithiasis in adults: a systematic review for an American College of Physicians. Clinical guideline. Ann Intern Med 2013; 158(7): 535-43.

2. Marangella M, Vitale C, Petrarulo M, Rovera L, Dutto F. Effects of mineral composition of drinking water on risk for stone formation and bone metabolism in idiopathic calcium nephrolithiasis. Clin Sci 1996; 91: 313-8.

3. Hosking DH, Erickson SB, Van den Berg CJ, Wilson DM, Smith LH. The stone clinic effect in patients with idiopathic calcium urolithiasis. J Urol 1983; 130: 1115-8.

4. Preminger GM. The metabolic evaluation of patients with recurrent nephrolithiasis: a review of comprehensive and simplified approaches. J Urol 1989; 141: 760-3. 